\title{
Risk of pressure injuries in fully dependent older people during the COVID-19 pandemic in Brunei
}

Nurizzqah Bungsu, Shyh Poh Teo

BRUNEI IS A SMALL COUNTRY in Southeast Asia that is experiencing a rapid increase in the proportion of older people. There are no residential care facilities; thus, older people, including those who are fully dependent, are cared for by their families. This is possible with the current societal structure of close extended families living together, but it will gradually be less feasible with the reducing fertility rate and household numbers reducing to individual nuclear families. Community nursing services visit fully dependent patients at home, mainly for indwelling catheter and nasogastric tube changes, as well as wound reviews. If a medical review is required, an ambulance is contacted to bring the patients to the nearest hospital. For patients with pressure injuries, family members or caregivers will be taught how to manage them in terms of wound dressings and pressure-relieving approaches, with intermittent nursing reviews to monitor for progress.

During the COVID-19 pandemic, there was an increase in the number of older people admitted to hospital with severe pressure injuries. This was likely partly due to social restrictions resulting in reduced caregivers and support services, compounded by limited follow-up from community nursing visits. ${ }^{1}$ Within six months, there were nine fully dependent older adults admitted with Stage 4 pressure injuries complicated by osteomyelitis. These patients were frail and malnourished, and the clinicians had concerns regarding the care that had been provided at home. The patients all had moderate-to-severe pain and experienced prolonged hospitalisation for intravenous antibiotics and wound management. Two-thirds died in hospital. ${ }^{2}$

A pressure injury occurs when the skin and underlying tissues are damaged because of restricted blood flow from constant external forces or pressure. This is more likely to happen in fully dependent people over bony areas, such as the sacrum and heel. Pressure injuries can result in pain, prolonged hospital admissions, impaired quality of life and increased morbidity and mortality. ${ }^{3}$ A study of medical inpatients showed that the prevalence of pressure injuries can be as high as $20.4 \%$ if pressure injury risk assessment and prevention are poor. Risk factors that should be identified and managed to mitigate this risk include sensory deficits, incontinence, malnutrition, inactivity, inability to change position, and friction and shear. ${ }^{4}$ Ongoing monitoring is required to ensure optimal management of these risk factors to reduce the risk of developing pressure injuries.
How could we have reduced this risk of pressure injuries during the pandemic? An increased awareness of pressure injuries is required among healthcare workers and family caregivers. A pilot study from Hong Kong showed that educational sessions and workshops for caregivers improved their knowledge and compliance with preventive measures. The content covered included risk factor identification, skin care and assessment, appropriate support surfaces and a demonstration of prevention approaches. ${ }^{5}$

Although there were limited visits from nurses and allied health professionals, each clinical or community encounter with immobile or dependent patients should be considered as a valuable opportunity to assess for the presence of pressure injuries and associated risk factors. These vulnerable older people should be identified and proactively checked to ensure their wellbeing is maintained. The need for regular turning and repositioning, offloading vulnerable areas and frequent inspection should be emphasised. The availability of basic necessities, especially food and required care essentials such as diapers, should be checked. It is also important to ensure that caregivers are provided with emotional support to maintain their caregiving duties and avoid burnout. The use of telemedicine or virtual consultations may be a useful alternative 
to consider for patient reviews if these cannot be done in person.

In summary, the COVID-19 pandemic has led to restrictions that may increase the risk of pressure injuries. Caregivers and healthcare workers should proactively look out for these complications in older people who are fully dependent.

First published 2 December 2021.

\section{Authors}

Nurizzqah Bungsu BSc, PAPRSB Institute of Health Sciences, Universiti Brunei Darussalam, Brunei Shyh Poh Teo MBChB, FRACP, Consultant Geriatrician and Physician, Department of Internal Medicine, Raja Isteri Pengiran Anak Saleha Hospital, Bandar Seri Begawan, Brunei

Competing interests: None.

Funding: None.

Provenance and peer review: Not commissioned, peer reviewed.

Citation: Bungsu N, Teo SP. Risk of pressure injuries in fully dependent older people during the COVID-19 pandemic in Brunei. Aust J Gen Pract 2021;50 Suppl 53. doi: 10.31128/AJGP-COVID-53.

Correspondence to:

shyhpoh.teo@moh.gov.bn

\section{References}

1. Sofian AlH, Md Jappar SAN, Amzizulfadzillah AF, et al. COVID-19 pandemic situation analysis for Brunei Darussalam - Perspectives from the front line, geriatric medicine and mental health. Pac J Med Sci 2021;21(2):5-11.

2. Teo SP, Halim N, Ahmad MH. Stage 4 sacral pressure injuries among fully dependent older adults during the COVID-19 pandemic. Aging Med Healthc 2021. doi: 10.33879/AMH. XXX.2021.03014 [ePub ahead of print].

3. Teo SP, Halim N. Pressure injury prevention and management - Hospital initiatives and interventions. J Gerontol Geriatr 2019;67:235-38.

4. Kula M, Teo SP. Prevalence and risk factor assessments of pressure ulcers among medical in-patients in RIPAS Hospital. Brunei Int Med J 2018;14:151-56.

5. Chong KW, Lee SW. A home-based education programme in pressure injury prevention and management for caregivers of elderly patients: A pilot study. Asian J Gerontol Geriatr 2017;12:53-59. 